\title{
Sugar Tech Special Issue: History of Sugarcane Breeding, Germplasm Development and Related Molecular Research
}

\author{
Prakash Lakshmanan $^{1,2,3}$ (1) Phillip Jackson ${ }^{4}$ G. Hemaprabha ${ }^{5}$ Yang Rui Li $^{1}$
}

Received: 24 November 2021 / Accepted: 24 November 2021/Published online: 14 December 2021

(C) The Author(s), under exclusive licence to Society for Sugar Research \& Promotion 2021

Varieties are the cornerstone of sugarcane production. Despite having a relatively short history, sugarcane breeding has made an impressive contribution to the expansion and profitability of sugar industries across the world. A large number of sugarcane breeding programs are now in operation in the majority of sugarcane producing countries. However, a comprehensive account of their course of development, breeding strategies and contributions at the country level is lacking. In this Special Issue a series of invited papers are presented that document the

Prakash Lakshmanan

plakshmanan2018@outlook.com; Prakash@gxaas.net

Phillip Jackson

phillip.jackson@csiro.au

G. Hemaprabha

ghemaprabha1@gmail.com

Yang Rui Li

liyr@gxaas.net

1 Key Laboratory of Sugarcane Biotechnology and Genetic Improvement (Guangxi), Ministry of Agriculture and Rural Affairs, Guangxi Key Laboratory of Sugarcane Genetic Improvement, Sugarcane Research Institute, Guangxi Academy of Agricultural Sciences, Nanning 530007, China

2 Interdisciplinary Research Centre for Agriculture Green Development in Yangtze River Basin, College of Resources and Environment, Southwest University, Chongqing 400716, China

3 Queensland Alliance for Agriculture and Food Innovation, University of Queensland, St Lucia, QLD 4067, Australia

4 CSIRO Agriculture and Food, Townsville, Qld 4814, Australia

5 ICAR-Sugarcane Breeding Institute, Coimbatore 641 007, India overall history and current status of sugarcane breeding and germplasm development worldwide. Papers from nearly all major sugarcane producing countries, as well as from many smaller producing countries which have important and technically advanced breeding programs (e.g., Japan, Reunion, Mauritius, Barbados, others) are presented. These papers were commissioned and written by leading sugarcane breeders and experts within each country. Each article provides key basic information about characteristics and status of the sugar industry in the country, a brief history of variety improvement, a list of institutions involved in sugarcane improvement, an outline of the objectives and methods being currently used, notes on the current major cultivars and research focus, germplasm collection and a commentary on future research directions. Besides the key theme reviews, articles reviewing several key technology areas important in sugarcane breeding, including germplasm development and utilization are also presented.

This is the first time that detailed and comparable information about sugarcane breeding from all the major sugarcane producing countries has been brought together. We hope this collation of reviews by leading sugarcane breeders and experts across the world will provide a useful reference source for the breeders, scientists, industry investors, technologists and students involved in sugarcane improvement programme all over the world generally. It is also hoped that this may in turn help stimulate useful exchange of ideas about directions of sugarcane improvement, germplasm (seeds and varieties), and collaboration across breeding programs worldwide.

Developing new profitable sugarcane varieties remains one of the highest priorities for sugarcane industry stakeholders in all countries. Experienced sugarcane growers and mill owners worldwide fully appreciate the large 
beneficial impacts that highly productive varieties can have on their business profitability. However, in the current changing adverse climate scenario all over the globe, many also realise the adverse, sometimes disastrous, impact when a widely adopted variety breaks down in the face of unforeseen pest and disease incursions or responds poorly to an extreme climatic event such as drought or flood. Because of this, access to a pipeline of new varieties from one or more effective breeding programs will remain important for the future development and sustainability of all sugarcane industries worldwide.

Unlike some other crops such as rice maize, wheat, soybean and cotton where large, competitive, multi-national breeding programs have evolved, sugarcane breeding worldwide to date has remained very partitioned on a national basis. Most sugarcane breeding programs worldwide are focused on only one country, and usually funded by national governments and/or local sugarcane industries. The scale of effort varies greatly between individual programs and different countries. Despite the differences, it is also striking that there are major basic similarities in breeding methodology among all programs currently. In fact, sugarcane cultivars throughout the world currently arise from breeding programs deploying a broad approach which have remained largely unchanged for many decades. This approach comprises a regular and continuous pipeline of operations of annual crossing among selected parent clones to produce large populations of seedling clones, followed by selection of superior clones in successive stages of field trials for usually 9-12 years for important traits.

The common basic approach persisting across all breeding programs perhaps reflects some level of conservatism in sugarcane breeding strategies worldwide. This may be regarded by some stakeholders as reflecting either, or both, strength and weakness. On the one hand, perhaps it is a strength because it indicates a robust and well-established methodology is being used. On the other hand, some may regard it as a weakness because it reflects an absence of effective innovation and technological "disruption", despite increasing concerns about declining rates of genetic gain by industry investors in comparison with other crops, including the other sugar crop and sugarcane's main competitor, sugar beet. It is also apparent that there is relatively little successful and routine application of modern molecular technologies for the commercial cultivar development, including breeding biotic and abiotic stress resistant varieties.
The exchange of varieties between countries is important to global sugarcane improvement. In fact, sugarcane varieties currently grown commercially in all countries arise due to international exchange and collaboration: all are derived from important ancestral parental material originally developed in a few countries. In particular, the contribution of parental clones originally bred and selected in India (Co- clones) and Indonesia (POJ- clones) is well known to all sugarcane breeders, and this is reinforced in some of the following papers. In some programs, efforts have been maintained over time in accessing the best varieties from other countries, and this has usually paid good dividends. While very few varieties imported from other countries perform well directly as commercial cultivars (although occasionally some are successful), many make important contributions as parents to local breeding programs, especially in combinations with locally adapted germplasm. However, despite the potential value of international variety exchange, anecdotal information suggests that in many countries relatively little effort in recent decades has been devoted to accessing the best varieties and parents from programs worldwide, with most focus on locally selected parents. In some cases, restrictions on importing germplasm arise because of biosecurity concerns, coupled with lack of quarantine facilities and expertise. However, with the development of commercial quarantine services (e.g., Visacane at CIRAD, France) such concerns are now able to be readily addressed.

The last decade has witnessed extraordinary technological advances that are likely to accelerate the rates of genetic gain in all crops, and sugarcane is no exception. This includes decoding the genome of sugarcane, targeted genome editing, application of genomic selection and phenomics for breeding, and progress in data science. Exploiting these technologies for speed breeding of sugarcane remains a challenge but also presents a great opportunity.

We hope that this Special Issue will serve as a valuable reference for sugarcane breeding, and facilitates an exchange of information and ideas about the current status of sugarcane breeding and breeding innovations to harness the full potential of this remarkable crop.

Publisher's Note Springer Nature remains neutral with regard to jurisdictional claims in published maps and institutional affiliations. 


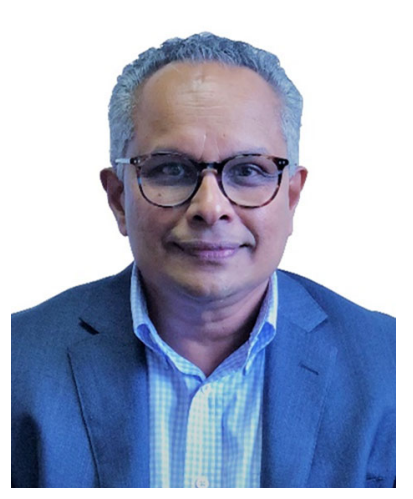

Prakash Lakshmanan is a sugarcane scientist with more than thirty years of research and research management experience in academia and industry research and development institutions. Dr Lakshmanan started his career as a Research Scientist in Thehans Laboratories, a plant biotechnology company in Singapore in 1989 before joining the faculty of Department of Biosciences at The National University of Singapore in 1992. In 1998, he joined Bureau of Sugar Experiment Stations (BSES) in Australia, one of the oldest and well-known sugarcane research institutes in the world, and led research focusing on trait development for variety improvement. Currently Dr Lakshmanan is Director, Sugarcane Research Institute, Nanning, China and Professor at Southwest University, Chongqing, China. He is also an Honorary Professor at the University of Queensland, Australia, and Editorial Manager (Sugarcane) of Sugar Tech journal.

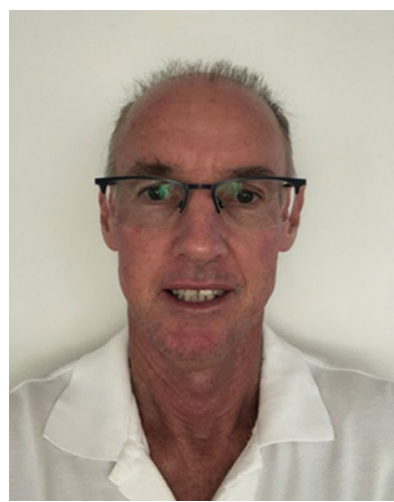

Phil Jackson was a scientist for 29 years with the Commonwealth Scientific and Industrial Research Organisation (CSIRO), Australia, leading sugarcane breeding programs in partnership with former Australian companies CSR Ltd and BSES Limited releasing $>20$ cultivars to the Australian sugarcane industry. During this time, he also conducted a range of research projects to improve the process of sugarcane breeding, particularly in areas of optimising selection systems in sugarcane breeding programs, economic assessment of traits, applying DNA markers to sugarcane breeding, utilizing diverse germplasm for sugarcane improvement, and breeding for water stress environments. He has led international collaborative projects in sugarcane breeding, and was Chairman of the International Society of Sugarcane Technologists Standing Committee on Germplasm and Breeding. He retired from a position as Senior Principal Research Scientist with CSIRO in 2017. He is a Life Member of the Australian Society of Sugarcane Technologists, and an Honorary Fellow with CSIRO.

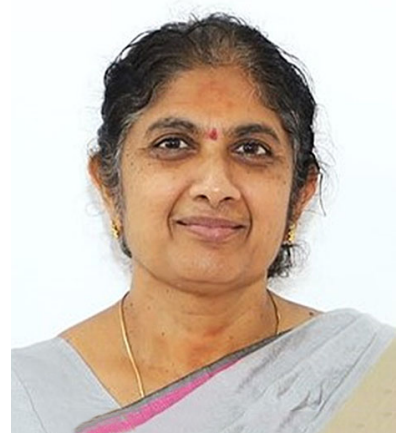

G. Hemaprabha is a sugarcane breeder with 35 years of experience and is presently the Acting Director of the ICAR Sugarcane Breeding Institute (SBI) located at Coimbatore, India, a historically important institution for pioneering sugarcane breeding and producing the initial interspecific hybrids of modern sugarcane. She has developed and released $25 \mathrm{sug}$ arcane varieties, which made a significant impact in both tropical and subtropical India. Her work on introgression breeding has provided a clear understanding on the behaviour of interspecific hybrids involving different species for economic traits including climate resilience. Dr Hemaprabha has developed a comprehensive database of Coimbatore canes (Co canes) bred over a century of breeding at SBI, Coimbatore, which has great practical value in sugarcane breeding. Her contributions to the development and application of sugarcane-specific STMS markers, genomic selection and true seed development are expected to improve precision and efficiency of sugarcane breeding and production. She has won several academic and industry awards.

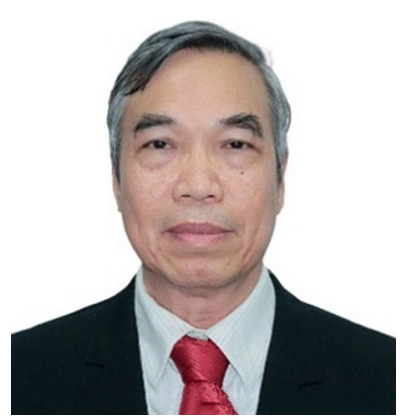

Yang Rui $\mathbf{L i}$ is a sugarcane specialist with 34 years of teaching, research and academic management experience in universities and research institutions. He is a Professor and was Vice- President of Guangxi University and President of Guangxi Academy of Agricultural Sciences. Dr. Li is now Lifetime Professor of Guangxi and Director of Sugarcane Research Center, Chinese Academy of Agricultural Sciences, President of International Association for Professionals in Sugar and Integrated Technologies (IAPSIT) and Vice-President of Society for Sugar Research and Promotion (SSRP). Dr. Li's research interests include sugarcane germplasm innovation and utilization, sugarcane farming science and technologies, sugarcane breeding, sugarcane functional genomics and proteomics, and sugarcane biological nitrogen fixation. Dr Li led the sugarcane breeding and farming technology research in Guangxi science 1998, and has been serving as the Chief Expert of the National Joint Research Program for Elite Sugarcane Variety Development in China since 2018. Dr. Li won the Lifetime Achievement Awards from SSRP in 2011 and IAPSIT in 2014, respectively. 